\title{
2D mapping of ice species in molecular cores*
}

\section{Jennifer A. Noble ${ }^{1}$, H. J. Fraser ${ }^{1}$, K. M. Pontoppidan ${ }^{2}$, Y. Aikawa ${ }^{3}$ and I. Sakon ${ }^{4}$}

${ }^{1}$ Department of Physics, University of Strathclyde, 107 Rottenrow, Glasgow G4 0NG, Scotland email: jennifer.noble@strath.ac.uk

${ }^{2}$ California Institute of Technology, Division of Geological and Planetary Sciences, MS 150-21, Pasadena, CA 91125, U.S.A.

${ }^{3}$ Department of Earth and Planetary Sciences, Kobe University, Kobe 657-8501, Japan

${ }^{4}$ Department of Astronomy, Graduate School of Science, University of Tokyo, 7-3-1 Hongo, Bunkyo-ku, Tokyo 113-0003, Japan

Abstract. We present data from our ice mapping program IMAPE on the AKARI satellite. Initial results show a correlation between the abundance of $\mathrm{CO}_{2(s)}$ and $\mathrm{H}_{2} \mathrm{O}_{(s)}$, consistent with previous studies. We can trace abundances of molecules across a core using a single observation.

Keywords. astrochemistry, ISM: clouds, ISM: molecules, techniques: spectroscopic

\section{Introduction}

In dense cores, much of the molecular material is frozen on the surface of dust grains. AKARI allows the simultaneous observation of multiple lines of site (los) through a core. We observed a 1'x1' region towards 20 cores, between 2.5-5.0 $\mu \mathrm{m}$. Data was reduced using our own pipeline (Noble et al. in prep.), producing spectra for 31 los.

\section{Results and Conclusions}

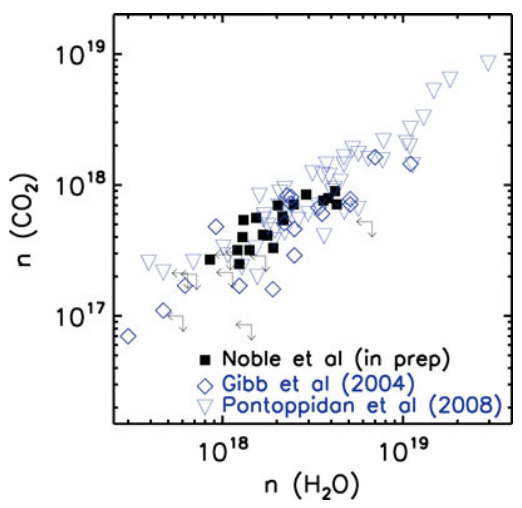

Figure 1. Correlation plot of $n\left(\mathrm{CO}_{2}\right)$ vs $n\left(\mathrm{H}_{2} \mathrm{O}\right)$ for 31 los in the AKARI IMAPE program.

The abundance of $\mathrm{H}_{2} \mathrm{O}$ and $\mathrm{CO}_{2}$ was calculated for each los using laboratory data, and is presented in Figure 1. Abundances agree with previous studies (as shown in Figure 1) and a clear correlation is seen between $n\left(\mathrm{H}_{2} \mathrm{O}\right)$ and $n\left(\mathrm{CO}_{2}\right)$ in the cores observed.

\section{References}

Gibb, E. L. et al. 2004, ApJS, 151, 35

Pontoppidan, K. M. et al. 2008, ApJ, 678, 1005

* Based on observations with AKARI, a JAXA project with the participation of ESA. 